\title{
Synchronization of Delayed Reaction-diffusion Neural Networks with Markovian Jumping Parameters
}

\author{
Jianping Zhou \\ Department of Automation, Nanjing University of \\ Science and Technology, Nanjing 210094, China \\ School of Computer Science, Anhui University of \\ Technology, Ma'anshan 243002, China
}

\author{
Hao Shen \\ School of Electrical Engineering \& Information, Anhui \\ University of Technology, \\ Ma'anshan 243002, China
}

\author{
Zhen Wang \\ College of Information Science and Engineering, \\ Shandong University of Science and Technology, \\ Qingdao 266510,China
}

\begin{abstract}
This paper deals with the drive-response type synchronization of delayed reaction-diffusion neural networks with Markovian jumping parameters. In terms of linear matrix inequalities, a sufficient condition is proposed to ensure the drive system and the response system to be stochastically synchronized. An example is provided to demonstrate the effectiveness of the proposed result.
\end{abstract}

Keywords- Neural networks, Reaction-diffusion, Markovian jumping parameters, Synchronization

\section{INTRODUCTION}

During the past decades, synchronization of neural networks with or without time delays has received considerable attention due to its potential application in the areas of secure communication, parallel recognition, and associative memory $[1,2]$. As is known to all, the latching phenomenon usually happens in neural networks, which can be dealt with effectively by extracting finite state representation from trained network. In other words, the neural networks may have finite modes and the mode may jump from one to another at different times. The jumping between different modes can be governed by a Markov chain [3-6]. Therefore, it is necessary to study the synchronization problem for neural networks with Markovian jumping parameters. On the other hand, in electronic implementation of neural networks, the density of the electromagnetic field is generally asymmetrical, which will lead to reactiondiffusions [7]. Thus, much effort has been devoted to the study of the synchronization of reaction-diffusion neural networks in the past few years [8-11]. According to the authors' knowledge, however, for the synchronization of reaction-diffusion neural networks with Markovian jumping parameters, no results have been reported so far.

In this paper, we are concerned with the drive-response type synchronization of delayed reaction-diffusion neural networks with Markovian jumping parameters. The jumping parameters considered here are generated from a continuoustime discrete-state homogeneous Markov process, which is governed by a Markov process with finite state space. Based on the Lyapunov functional method, a sufficient condition is presented, which ensures the drive system and the response system to be stochastically synchronized. The criterion is expressed as a set of linear matrix inequalities (LMIs), and thus can be easily checked by using standard numerical software Matlab. In order to illustrate the effectiveness of the proposed condition, a numerical example is given.

\section{PROBLEM FORMULATION}

Consider the following delayed reaction-diffusion neural network with Markovian jumping parameters:

$$
\left\{\begin{aligned}
\frac{\partial x(t, z)}{\partial t}= & D \circ \nabla^{2} x(t, z)-C\left(r_{t}\right) x(t, z)+A\left(r_{t}\right) f(x(t, z)) \\
& +B\left(r_{t}\right) f(x(t-\tau(t), z))+J, \\
x(t, z)= & \phi(t, z), \quad(t, z) \in[-\tau, 0] \times \Omega, \\
x(t, z)= & 0, \quad(t, z) \in[-\tau,+\infty) \times \partial \Omega,
\end{aligned}\right.
$$

where $x(t, z)=\left[x_{1}(t, z) \cdots x_{n}(t, z)\right]^{T}$ is the state vector; $f(x)=\left[f_{1}\left(x_{1}\right) \cdots f_{n}\left(x_{n}\right)\right]^{T}$ is the activation function which satisfies the Lipschitz condition, i.e., there exists constants $F_{i}>0, i=1, \cdots, n$, such that

$$
\left|f_{i}\left(\varepsilon_{2}\right)-f_{i}\left(\varepsilon_{2}\right)\right| \leq F_{i}\left|\varepsilon_{2}-\varepsilon_{1}\right|,
$$

for any $\varepsilon_{1}, \varepsilon_{1} \in R ; \phi(t, z)$ is a continuous vector function; $\nabla^{2}=\sum_{l=1}^{m} \partial^{2} / \partial z_{l}^{2}$ is the Laplace operator; $A\left(r_{t}\right)=\left(a_{i j}\left(r_{t}\right)\right)_{n \times n}$, $B\left(r_{t}\right)=\left(b_{i j}\left(r_{t}\right)\right)_{n \times n}, C\left(r_{t}\right)=\operatorname{diag}\left(c_{1}\left(r_{t}\right), \cdots, c_{n}\left(r_{t}\right)\right)>0$, and $D=\operatorname{diag}\left(d_{1}, \cdots, d_{m}\right)>0$ are, respectively, the connection weight matrix, the delayed connection weight matrix, the charge rate matrix, and the diffusion rate matrix; $J=\left[J_{1} \cdot \cdots J_{n}\right]^{T}$ is a constant bias vector; $\Omega=\left\{z=\left[z_{1} \cdots z_{m}\right]^{T} \mid \varsigma_{1} \leq z_{l} \leq \varsigma_{2}, \varsigma_{1}<\varsigma_{2}, l=1, \cdots ; m\right\}$ is a compact set in $R^{m}$ with smooth boundary $\partial \Omega .\left\{r_{t}\right\}$ is a continuous-time Markovian process with right continuous trajectories and taking values in a finite set $S=\{1, \cdots, s\}$ with transition probability matrix $\Pi=\left\{\pi_{i j}\right\}$ given by

$$
\operatorname{Pr}\left\{r_{t+h}=j / r_{t}=i\right\}=\left\{\begin{array}{l}
\pi_{i j} h+o(h), \quad i \neq j, \\
1+\pi_{i i} h+o(h), \quad i=j,
\end{array}\right.
$$

where $h>0, \quad \lim _{h \rightarrow 0} o(h) / h=0, \pi_{i j} \geq 0(i \neq j) \quad$ is the transition rate from mode $i$ at time $t$ to mode $j$ at time 
$t+h, \quad$ and $\pi_{i i}=-\sum_{j=1, j \neq i}^{s} \pi_{i j}$. Note that the boundary conditions are chosen to be of the Dirichlet type as in [12].

In the paper, system (1) is considered as a drive system. The response system is given by

$$
\left\{\begin{aligned}
& \frac{\partial y(t, z)}{\partial t}= D \circ \nabla^{2} y(t, z)-C\left(r_{t}\right) y(t, z)+A\left(r_{t}\right) f(y(t, z)) \\
&+B\left(r_{t}\right) f(y(t-\tau(t), z))+J+U, \\
& y(t, z)= \varphi(t, z), \quad(t, z) \in[-\tau, 0] \times \Omega, \\
& y(t, z)=0, \quad(t, z) \in[-\tau,+\infty) \times \partial \Omega,
\end{aligned}\right.
$$

where $y(t, z)$ is the state vector of the response system, $\varphi(t, z)$ is a continuous vector function, $U$ is the state feedback controller to be designed later.

Let $e(t, z)=y(t, z)-x(t, z)$ be the synchronization error. Then, the error dynamical system can be described by

$$
\left\{\begin{aligned}
\frac{\partial e(t, z)}{\partial t}= & D \circ \nabla^{2} e(t, z)+\left[K_{1}\left(r_{t}\right)-C\left(r_{t}\right)\right] e(t, z)+A\left(r_{t}\right) g(e(t, z)) \\
& +K_{2}\left(r_{t}\right) e(t-\tau(t), z)+B\left(r_{t}\right) g(e(t-\tau(t), z)), \\
e(t, z)= & \varphi(t, z)-\phi(t, z), \quad(t, z) \in[-\tau, 0] \times \Omega, \\
\mathrm{e}(t, z)= & 0, \quad(t, z) \in[-\tau,+\infty) \times \partial \Omega,
\end{aligned}\right.
$$

if the controller

$$
U=K_{1}\left(r_{t}\right) e(t, z)+K_{2}\left(r_{t}\right) e(t-\tau(t), z),
$$

where $K_{1}\left(r_{t}\right)$ and $K_{2}\left(r_{t}\right)$ are the feedback gain matrices. In (3), $g(e(\cdot, z))=f(e(\cdot, z)+x(\cdot, z))-f(x(\cdot, z))$. Note that the following condition holds

$$
e^{T}(t, z) F H F e(t, z)-g^{T}(t, z) H g(t, z) \geq 0,
$$

for any $n$-dimensional positive definite diagonal matrix $H$ Here $F=\operatorname{diag}\left(F_{1}, \cdots, F_{n}\right)$.

Definition 1. System (1) and system (2) are said to be stochastically synchronized if there exists a positive constant $\rho_{0}$, such that

$$
E \int_{\Omega} e^{T}(s, z) e(s, z) d z \leq \rho_{0}
$$

for any initial mode $r_{0}$, where $E\{\cdot\}$ denotes the expectation operator.

Lemma $1[12]$. If $e(t, z)$ is a solution of system (3), then for any $i \in\{1, \cdots, n\}$ and $l \in\{1, \cdots m\}$,

$$
-\int_{\varsigma_{1}}^{\varsigma_{2}}\left(\frac{\partial e_{i}(t, z)}{\partial z_{l}}\right)^{2} d z_{l} \leq-\frac{\pi^{2}}{\left(\varsigma_{2}-\varsigma_{1}\right)^{2}} \int_{\varsigma_{1}}^{\varsigma_{2}} e_{i}^{2}(t, z) d z_{l} .
$$

\section{SYNCHRONIZATION CONDITION}

Theorem 1. System (1) and system (2) are stochastically synchronized if there exist positive definite diagonal matrices $H, P_{i}=\operatorname{diag}\left(P_{i 1}, \cdots, P_{i n}\right), \quad i=1, \cdots, s \quad$ and $n$ - dimensional positive definite matrices $Q_{1}, Q_{2}, G_{1 i}, G_{2 i}, i=1, \cdots, s$, such that

$$
\Psi_{i}=\left[\begin{array}{cccc}
\Gamma & G_{2 i} & P_{i} A_{i} & P_{i} B_{i} \\
G_{2 i}^{T} & -(1-\eta) Q_{1} & 0 & 0 \\
A_{i}^{T} P_{i} & 0 & Q_{2}-H & 0 \\
B_{i}^{T} P_{i} & 0 & 0 & -(1-\eta) Q_{2}
\end{array}\right]<0,
$$

where

$$
\begin{aligned}
\Gamma_{i}= & 2 P_{i}\left[-C_{i}-m \pi^{2} D /\left(\varsigma_{2}-\varsigma_{1}\right)^{2}\right]+G_{1 i}+G_{1 i}^{T} \\
& +\sum_{j=1}^{s} \pi_{i j} P_{j}+Q_{1}+F H F .
\end{aligned}
$$

Moreover, the gain matrices are given by

$$
K_{1 i}=P_{i}^{-1} G_{1 i}, K_{2 i}=P_{i}^{-1} G_{2 i}, \quad i=1, \cdots, s \text {. }
$$

Proof. Consider the following Lyapunov functional:

$$
\begin{aligned}
V\left(e(t, z), t, r_{t}\right) & =\int_{\Omega} e(t, z)^{T} P\left(r_{t}\right) e(t, z) d z \\
& +\int_{\Omega} \int_{t-\tau(t)}^{t} e^{T}(v, z) Q_{1} e(v, z) d v d z . \\
& +\int_{\Omega} \int_{t-\tau(t)}^{t} g^{T}(e(v, z)) Q_{2} g(e(v, z)) d v d z .
\end{aligned}
$$

Let $L$ be the weak infinitesimal generator of the random process $\left\{e(t, z), t, r_{t}\right\}$. Then, for each $r_{t}=i, i \in S$, we have

$$
\begin{aligned}
& L V\left(e(t, z), t, r_{t}\right) \\
& \leq \sum_{j=1} \pi_{i j} \int_{\Omega} e^{T}(t, z) P_{j} e(t, z) d z+\int_{\Omega} 2 e(t, z)^{T} P_{i} D \circ \nabla^{2} e(t, z) d z \\
& \quad+\int_{\Omega}\left(e^{T}(t, z) P_{i}\left[K_{1 i}-C_{i}\right] e(t, z)+e^{T}(t, z)\left[K_{1 i}-C_{i}\right]^{T} P_{i}^{T} e(t, z)\right) d z \\
& \quad+\int_{\Omega}\left(e^{T}(t, z) P_{i} A g(e(t, z))+g^{T}(e(t, z)) A_{i}^{T} P_{i} e(t, z)\right) d z \\
& \quad+\int_{\Omega}\left(e^{T}(t, z) P_{i} K_{2 i} e(t-\tau(t), z)+e^{T}(t-\tau(t), z) K_{2 i}^{T} P_{i} e(t, z)\right) d z \\
& \quad+\int_{\Omega}\left(e ^ { T } ( t , z ) P _ { i } B _ { i } g \left(e(t-\tau(t), z)+g^{T}\left(e(t-\tau(t), z) B_{i}^{T} P_{i} e(t, z)\right) d z\right.\right. \\
& \quad+\int_{\Omega} e^{T}(t, z) Q_{e} e(t, z)-(1-\eta) e^{T}(t-\tau(t), z) Q e(t-\tau(t), z) d z \\
& \quad+\int_{\Omega} g^{T}(e(t, z)) Q_{2} g(e(t, z))-(1-\eta) g^{T}(e(t-\tau(t), z)) Q_{2} g(e(t-\tau(t), z)) d z .
\end{aligned}
$$

Using the integration by parts formula in the $z_{l}$ - direction, we obtain

$$
\int_{\varsigma_{1}}^{\varsigma_{2}} e_{i}(t, z) \frac{\partial^{2} e_{i}(t, z)}{\partial z_{l}^{2}} d z_{l}=-\int_{\varsigma_{1}}^{\varsigma_{2}}\left(\frac{\partial e_{i}(t, z)}{\partial z_{l}}\right)^{2} d z_{l}
$$

By Lemma 1, it can be seen that

$$
\int_{\varsigma_{1}}^{\varsigma_{2}} e_{i}(t, z) \frac{\partial^{2} e_{i}(t, z)}{\partial z_{l}^{2}} d z_{l} \leq-\frac{\pi^{2}}{\left(\varsigma_{2}-\varsigma_{1}\right)^{2}} \int_{\varsigma_{1}}^{\varsigma_{2}} e_{i}^{2}(t, z) d z_{l} .
$$

Integrating both sides of (7) with respect to $z_{1}, \cdots, z_{l-1}, z_{l+1}, \cdots, z_{m}$ in $\Omega$, we have

$$
\int_{\Omega} e_{i}(t, z) \frac{\partial^{2} e_{i}(t, z)}{\partial z_{l}^{2}} d z \leq-\frac{\pi^{2}}{\left(\varsigma_{2}-\varsigma_{1}\right)^{2}} \int_{\Omega} e_{i}^{2}(t, z) d z .
$$

It follows that

$$
\begin{aligned}
& \int_{\Omega} 2 e^{T}(t, z) P_{i} D \nabla^{2} e(t, z) d z \\
& \quad \leq-\frac{m \pi^{2}}{\left(\varsigma_{2}-\varsigma_{1}\right)^{2}} \int_{\Omega} e^{T}(t, z)\left(P_{i} D+D P_{i}\right) e(t, z) d z .
\end{aligned}
$$

Let 


$$
\vartheta=\left[e^{T}(t, z) e^{T}(t-\tau(t), z) g^{T}(e(t, z)) g^{T}(e(t-\tau(t), z))\right]^{T} .
$$

By (4), (6), (8), we get

$$
L V\left(e(t, z), t, r_{t}\right) \leq \int_{\Omega} \vartheta^{T} \Psi_{i} \vartheta d z
$$

Let

$$
\lambda_{0}=\min \left\{\lambda_{\min }\left(-\Psi_{i}\right), i \in S\right\}
$$

where $\lambda_{\text {min }}$ denotes the minimum eigenvalue of $\Psi_{i}$. Then, By Dynkin's formula [13] and (9), we have

$$
\begin{aligned}
E\left\{V\left(e(t, z), t, r_{t}\right)\right\} & -E\left\{V\left(e(0, z), 0, r_{0}\right)\right\} \\
& \leq E \int_{0}^{t} L V\left(e(t, z), t, r_{t}\right) d s \\
& \leq-\lambda_{0} E \int_{\Omega} e^{T}(s, z) e(s, z) d z .
\end{aligned}
$$

Since $E\left\{V\left(e(t, z), r_{t}\right)\right\} \geq 0$, it follows that

$$
E \int_{\Omega} e^{T}(s, z) e(s, z) d z \leq \frac{1}{\lambda_{0}} E\left\{V\left(e(0, z), 0, r_{0}\right)\right\}, \quad t \geq 0 .
$$

that is, system (1) and system (2) are stochastically synchronized.

Remark 1. Theorem 1 gives a sufficient condition to ensure the stochastic synchronization of the delayed reaction-diffusion neural networks with Markovian jumping parameters. The condition, which is expressed as a set of linear matrix inequalities (LMIs), can be easily checked by using standard numerical software Matlab.

\section{NUMERICAL EXAMPLE}

Consider a drive-response system described by system (1) and (2), where

$$
\begin{gathered}
A_{1}=\left[\begin{array}{cc}
0.15 & 0.01 \\
-0.27 & -0.18
\end{array}\right], A_{2}=\left[\begin{array}{cc}
0.89 & -0.80 \\
0.56 & -0.71
\end{array}\right], \\
B_{1}=\left[\begin{array}{cc}
-0.32 & -0.45 \\
0.29 & 0.77
\end{array}\right], B_{2}=\left[\begin{array}{cc}
-0.53 & 0.12 \\
-0.11 & -0.39
\end{array}\right], \\
C_{1}=\left[\begin{array}{cc}
0.56 & 0 \\
0 & 0.81
\end{array}\right], C_{2}=\left[\begin{array}{cc}
1.10 & 0 \\
0 & 0.37
\end{array}\right], \\
D=\left[\begin{array}{cc}
0.009 & 0 \\
0 & 0.012
\end{array}\right], \Pi=\left[\begin{array}{cc}
-3 & 3 \\
0.6 & -0.6
\end{array}\right], \\
f(x)=\left[\tanh \left(x_{1}\right) \tanh \left(x_{2}\right)\right]^{T}, J=\left[\begin{array}{ll}
0.93 & 0.71
\end{array}\right]^{T} \\
\tau(t)=0.5, m=2, \varsigma_{1}=0.02, \varsigma_{2}=0.05 .
\end{gathered}
$$

Obviously, $\eta=0$ and $F$ can be taken as $F=\operatorname{diag}(1,1)$. Employing the Matlab LMI Toolbox to solve the LMIs in (5), we can obtain

$$
\begin{aligned}
& H=\operatorname{diag}(1.5689,1.5684), \\
& P_{1}=P_{2}=\operatorname{diag}(0.0142,0.0107), \\
& Q_{1}=\operatorname{diag}(1.0154,1.0150), \\
& Q_{2}=\operatorname{diag}(0.8303,0.8301), \\
& G_{11}=\operatorname{diag}(1.0187,1.0174), \\
& G_{12}=\operatorname{diag}(1.0185,1.0175) \\
& G_{21}=G_{22}=\operatorname{diag}(0.3379,0.3379)
\end{aligned}
$$

Thus, by Theorem 1, it can be concluded that System (1) and system (2) are stochastically synchronized when the control gain matrices are given by

$$
\begin{gathered}
K_{11}=\operatorname{diag}(71.5327,95.3482), \\
K_{12}=\operatorname{diag}(71.7202,95.1915), \\
K_{21}=K_{22}=\operatorname{diag}(23.7936,31.6138) . \\
\text { V. } \quad \text { CONCLUSIONS }
\end{gathered}
$$

\section{CONCLUSIONS}

In this paper, we have dealt with the problem of driveresponse type synchronization for a class of neural networks, which include time-delays, Markovian jumping parameters, and reaction-diffusions. A sufficient condition which ensures the drive system and the response system to be stochastically synchronized has been proposed in terms of LMIs. In order to illustrate the effectiveness of the proposed condition, a numerical example has been provided. It should be noted that the transition probabilities considered here are assumed to be completely known in order to facilitate research. As noted by Zhang and Boukas [14], however, obtaining the ideal information on all transition probabilities is questionable or generally expensive. Thus, one of our future research directions will be further investigate the synchronization problem of the networks with partially unknown transition probabilities.

\section{REFERENCES}

[1] YU Wen-wu; Cao Jin-de. Synchronization Control of Stochastic Delayed Neural Networks. Physica A [J], 2007.373, PP: 252-260.

[2] HUANG Xia; LAM James; CAO Jin-de; Xu Sheng-yuan. Robust Synchronization Criteria for Recurrent Neural Networks via Linear Feedback. International Journal of Bifurcation and Chaos [J], 2007.17, PP: 2723-2738.

[3] WANG Lin-shan; ZHANG Zhe; WANG Yang-fan. Stochastic Exponential Stability of the Delayed Reaction-diffusion Recurrent Neural Networks with Markovian Jumping Parameters. Physics Letters A [J], 2008.372, PP: 3201-3209.

[4] SHEN Hao; XU Sheng-yuan; ZHOU Jian-ping. Fuzzy H $\infty$ filtering for nonlinear Markovian jump neutral delayed systems. International Journal of Systems Science [J], 2011.42, PP: 767-780.

[5] ZHAO Huan-yu; XU Sheng-yuan; ZOU Yun. Robust $\mathrm{H} \infty$ filtering for uncertain Markovian jump systems with mode-dependent distributed delays. International Journal of Adaptive Control and Signal Processing [J]. 2010. 24, PP: 83-94.

[6] MA Qian; XU Sheng-yuan; ZOU Yun; Lu Jin-jun. Stability of stochastic Markovian jump neural networks with mode-dependent delays. Neurocomputing [J], 2011, 74, PP: 2157-2163

[7] LIAO Xiao-xin; YANG Shu-zi; Cheng Shi-jie; Fu Yu-li. Stability of General Neural Networks with Reaction-Diffusion. Science in China [J], 2001.44, PP: 389-395.

[8] LOU Xu-yang; CUI Bao-tong. Asymptotic Synchronization of A Class of Neural Networks with Reaction-diffusion Terms and Timevarying Delays. Computers and Mathematics with Applications [J], 2006.52, PP: 897-904.

[9] WANG Yan-yan; CAO Jin-de. Synchronization of A Class of Delayed Neural Networks with Reaction-diffusion Terms. Physics Letters A [J], 2007. 369, PP: 201-211.

[10] ZHAO Bi-rong; DENG Fei-qi. Adaptive Exponential Synchronization of Stochastic Delay Neural Networks with Reactiondiffusion. Lecture Notes in Computer Science [J], 2009. 5551, PP: 550-559. 
[11] LV Teng; YAN Ping. Exponential Synchronization of Delayed Fuzzy Cohen-Grossberg Neural Networks with Reaction Diffusion Term. Lecture Notes in Computer Science [J], 2010. 6319, PP: 57-63.

[12] ZHOU Jian-ping; XU Sheng-yuan; SHEN Hao. Finite-time Robust Stochastic Stability of Uncertain Stochastic Delayed Reactiondiffusion Genetic Regulatory Networks. Neurocomputing [J], 2011.74, PP: 2790-2796.
[13] CAO Yong-yan; LAM James. Robust $\mathrm{H} \infty$ control of uncertain Markovian jump systems with time-delay. IEEE Transactions on Automatic Control [J]. 2000.45, PP: 77-83.

[14] ZHANG Li-xian; BOUKAS El-kebir (2009). Mode-dependent Ho filtering for discrete-time Markovian jump linear systems with partly unknown transition probabilities. Automatica [J]. 2009. 45, PP:14621467. 